\title{
BMJ Open Effect of sedation with inhaled anaesthetics on cognitive and psychiatric outcomes in critically ill adults: a systematic review protocol
}

\author{
Sean Cuninghame (D) , ${ }^{1}$ Kevin Gorsky (D) , ${ }^{2}$ Conall Francoeur, ${ }^{3}$ Davinia Withington, ${ }^{4}$ \\ Lisa Burry, ${ }^{5,6}$ Angela Jerath, ${ }^{2,7,8,9}$ Marat Slessarev ${ }^{10}$
}

To cite: Cuninghame S, Gorsky K, Francoeur C, et al. Effect of sedation with inhaled anaesthetics on cognitive and psychiatric outcomes in critically ill adults: a systematic review protocol. BMJ Open 2022;12:e052893. doi:10.1136/ bmjopen-2021-052893

- Prepublication history and additional supplemental material for this paper are available online. To view these files, please visit the journal online (http://dx.doi.org/10.1136/ bmjopen-2021-052893).

Received 28 April 2021 Accepted 18 January 2022

Check for updates

(C) Author(s) (or their employer(s)) 2022. Re-use permitted under CC BY-NC. No commercial re-use. See rights and permissions. Published by BMJ.

For numbered affiliations see end of article.

Correspondence to

Dr Marat Slessarev;

marat.slessarev@|hsc.on.ca

\section{ABSTRACT}

Introduction The COVID-19 pandemic has renewed interest in the use of inhaled anaesthetics for sedation of ventilated critically ill patients. Preliminary data show that inhaled anaesthetics reduce lung inflammation, time to extubation and intensive care unit length of stay compared with intravenous sedatives. However, the impact of inhaled anaesthetics on cognitive and psychiatric outcomes is not well described in this setting. Randomised controlled trials are underway to establish if inhaled anaesthetics affect these and other patient and health system outcomes. Our aim is to summarise the known effects of inhaled sedatives on cognitive and psychiatric outcomes.

Methods and analysis In this systematic review, we will use MEDLINE, EMBASE, and PsycINFO to identify studies from 1970 to 2021 that assessed cognitive and psychiatric outcomes in critically ill adult patients sedated with inhaled anaesthetics. We will include case series, observational and cohort studies and randomised controlled trials. We will exclude case studies due to the heterogeneity of reporting in these studies. For randomised controlled trials comparing inhaled to intravenous sedation, we will report cognitive and psychiatric outcomes for both study arms. Studies will be selected based on the Preferred Reporting Items for Systematic Reviews and Meta-Analyses checklist. Data will be extracted using a standardised data extraction tool by two independent reviewers. Studies will be assessed for bias using the Cochrane risk of bias tool for randomised controlled trials, or the Newcastle-0ttawa Scale for cohort and case-control studies. Findings will be reported according to outcome and descriptive statistics will be used to illustrate findings in a narrative fashion. Ethics and dissemination The systematic review uses published data and therefore does not require ethics approval. Results will be disseminated via publication in peer-reviewed journals and presentation at conferences related to the field.

PROSPERO registration number CRD42021236455.

\section{INTRODUCTION}

The SARS-CoV-2 2019 (COVID-19) pandemic and surge of critically ill patients has renewed interest in the use of inhaled anaesthetics for sedation for those with severe respiratory failure requiring mechanical ventilation.
Strengths and limitations of this study

To the best of our knowledge, this is the first systematic review examining the effects on volatile anaesthetics on cognition and psychiatric outcomes in critically ill adults.

- Important review as cognitive and psychiatric outcomes are important patient-centred outcomes and a number of randomised controlled trials are currently underway examining the effect of inhaled anaesthetics on physiological, patient and health system outcomes in patients with COVID-19 and non-COVID-19 respiratory failure.

- Limited exclusion criteria will allow for the maximum of papers on this topic to be included in our analysis.

- We expect few, if any, randomised controlled trials specifically analysing cognitive and psychiatric outcomes, and the review will likely be made up mostly of case series and observational studies.

Inhaled anaesthetics could address the shortages of intravenous sedative encountered during this pandemic because they can achieve the deep levels of sedation often required for patients with severe respiratory failure. ${ }^{1-4}$ Inhaled anaesthetics have additional benefits including reduced lung inflammation, improved oxygenation and are associated with shorter time to extubation and intensive care unit (ICU) length of stay. ${ }^{5-7}$ These are important considerations as the pandemic has put stress on the availability of critical care resources in many parts of the world ${ }^{8}$ However, there may be practical limitations to using inhaled anaesthetics in the ICU including equipment and properly trained personnel. In addition, some studies report various adverse effects of long-term inhaled anaesthetics such as dose-dependent respiratory depression, hypotension, malignant hyperthermia, diabetes insipidus and hepatitis, ${ }^{9}$ among others. ${ }^{10}$ Therefore, current randomised controlled trials will be 
helpful in establishing whether sedation with inhalational anaesthetics of critically ill patients with COVID-19 and other causes of respiratory failure is safe and improves patient and health system outcomes in comparison to traditional intravenous sedatives (eg, ClinicalTrials.gov, NCT04415060). While these trials will help to determine whether inhaled anaesthetics improve mortality and shorten duration of ventilation in patients with hypoxic respiratory failure, they will also assess the impact of inhaled sedatives on the quality-of-life measures after recovery from critical illness.

Cognitive impairment is common among critically ill patients and represents a spectrum of dysfunction from acute delirium to long-term cognitive impairment following discharge from the ICU. ${ }^{11}$ ICU delirium is a well-established acute entity that is associated with increased mortality, prolonged mechanical ventilation and hospitalisation, and increased healthcare costs. ${ }^{12} 13$ Furthermore, the presence of delirium while in the ICU may be a predictor of long-term cognitive disability, the incidence of which ranges from $10 \%$ to $58 \%$ across various studies. ${ }^{12} 14{ }^{15}$ While one systematic review did not find an association between sedative use and long-term cognitive dysfunction in ICU survivors, ${ }^{16}$ some prospective cohort studies found that intravenous sedative exposure in the ICU is associated with worse long-term cognitive function. ${ }^{15} 17$ However, a recent study found that when comparing intravenous sedation with a non-sedation approach in critically ill adults, there was no difference in cognition at 3 months following ICU discharge. ${ }^{18}$ There is also some evidence to suggest that intravenous sedation may contribute to psychiatric morbidity in the form of depression, anxiety and post-traumatic stress disorder (PTSD), which are also common among ICU survivors. ${ }^{19-22}$ While an association has been found between intravenous sedatives (ie, opioids, benzodiazepines and propofol) and the development of cognitive and psychiatric morbidity, the impact of inhaled anaesthetics on these outcomes has not been well described.

There is a clear association between critical illness and both acute and long-term cognitive dysfunction and psychiatric disturbances. Cognitive function and mental health are important determinants of academic and work success, levels of happiness and life expectancy. ${ }^{23-26}$ Given renewed interest in the use of inhaled anaesthetics for sedation of critically ill patients, it is imperative to ascertain the effect of these inhaled sedatives on cognitive and psychiatric outcomes. By providing an up-to-date summary and analysis of existing literature, this systematic review will lay the foundation for future research in this emerging field.

\section{AIMS}

The aim of this systematic review is to evaluate the impact of inhaled anaesthetics compared with intravenous sedatives on cognitive and psychiatric outcomes among mechanically ventilated critically ill adults. We will also

\begin{tabular}{ll} 
Table 1 Inclusion and exclusion criteria \\
\hline Population & $\begin{array}{l}\text { Adult ( }>18 \text { years old) patients } \\
\text { admitted to critical care units }\end{array}$ \\
Intervention & $\begin{array}{l}\text { Sedation with inhaled anaesthetics } \\
\text { Intravenous sedation }\end{array}$ \\
Comparator & $\begin{array}{l}\text { Short-term and long-term cognition } \\
\text { and psychiatric outcomes }\end{array}$ \\
\hline
\end{tabular}

investigate the methods used to measure and report cognitive and psychiatric morbidity in this setting.

\section{METHODOLOGY}

The Population-Intervention-Comparator-Outcome (PICO) strategy for this review is summarised in table 1.

\section{Types of studies}

Study designs will include case series, observational and cohort studies and randomised controlled trials. Case reports will be excluded given the often heterogenous nature of reporting results. We will consult with previous systematic reviews that have investigated inhaled anaesthetics use and assessed for cognitive performance after exposure while in the ICU to help identify primary literature on this topic. There is no restriction with respect to study sample size. Publication date will include studies published from 1970 until December 2020. Only English language studies will be used.

\section{Types of participants}

\section{Inclusion criteria}

- Adult (>18 years old) patients.

- Admitted to any ICU including general medicalsurgical, trauma and subspecialty ICUs (eg, cardiovascular, burns and neuro-based units).

- Patients who received inhaled sedatives for any period of time during their admission to an ICU. Treatment could be initiated in the ICU, the operating room or the emergency department.

- Treated with halothane, enflurane, isoflurane, sevoflurane or desflurane.

- Were assessed for cognitive and psychiatric (anxiety, depression, PTSD) outcomes using either subjective or objective tools at any stage during ICU admission or following ICU discharge.

\section{Exclusion criteria}

- Paediatric patients.

- Studies evaluating ether, trichloroethylene, methoxyflurane, xenon, nitrous oxide or other inhaled agents no longer relevant to modern clinical practice.

- Studies in language other than English.

\section{Types of intervention}

Interventions of interest will include exposure to inhaled anaesthetics (ie, sevoflurane, desflurane, isoflurane) for sedation in critically ill patients, and the concentration 
and duration of exposure to inhaled anaesthesia. Patients who received adjunctive intravenous sedation (eg, concomitant benzodiazepines or opioids) will be included as long as they satisfy the above inclusion criteria.

\section{Comparator}

While we expect few randomised controlled trials on this topic, our comparator for this systematic review will be any intravenous sedatives if the study design includes one.

\section{Types of outcome}

The primary outcomes of interest are subjective or objective measures of cognition, anxiety, depression or PTSD. Secondary outcomes are methods and instruments used to measure cognitive, anxiety, depression or PTSD outcomes (ie, subjective assessment vs objective testing; if objective, the specific test(s) used).

\section{Search strategy}

The search strategy will be performed and communicated in a way to maximise reproducibility of the search results and study conclusions following the Preferred Reporting Items for Systematic Reviews and MetaAnalyses (PRISMA) and Preferred Reporting Items for Systematic Reviews and Meta-Analyses search extension (PRISMA-S) guidelines. An initial search of Ovid MEDLINE will be performed in order to identify relevant Medical Subject Headings keywords in article titles and abstracts based on the PICO question outlined in table 1 . These keywords will then be included in a final expanded search of the MEDLINE, EMBASE, PsycINFO and the Cochrane Central Controlled Trials Register databases and a collection of their titles and abstracts uploaded into the online systematic review system, Covidence. We will include articles from 1970 to 2021. In addition, the reference sections of each of the included studies will also be reviewed in order to ensure all relevant articles are included. This includes all items discussed in the newly published PRISMA-S checklist for reporting specifically on the literature search component of systematic reviews. ${ }^{27}$ The full planned search strategy can be found in the online supplemental file 1 of this manuscript as part of completing the PRISMA-S checklist.

\section{Study selection}

Two independent reviewers will screen each article's title and abstract for inclusion in the final data extraction based on the aforementioned study inclusion/exclusion criteria using Covidence. In order to be considered for full-text review, screened articles will need to be identified for inclusion by both reviewers. Conflicts will be resolved by a third independent reviewer not involved in the initial screen. Full-text articles from the screening process will then be read for final inclusion in the review. A PRISMA flow diagram will be used to illustrate the number of studies screened, those chosen for inclusion, as well as reason(s) for exclusion from data extraction after full-text review. This diagram will be generated using
Covidence automatically after annotating specific studies for inclusion/exclusion.

\section{Data extraction}

Data extraction will be performed independently by two reviewers using Covidence's data extraction tool. We have created a data extraction tool and will test the tool on two papers prior to finalising the tool's parameters. Conflicts or missing data during data extraction are automatically flagged and will be resolved by a third reviewer when necessary. Data will be extracted with the following data points of interest:

1. Study characteristics-publication type, country, date of publication, study size and setting (ie, medical, surgical, cardiovascular, trauma centre, etc), industry sponsorship.

2. Population characteristics-age, sex, admission diagnosis, measurement of sedation, overall mortality, previous diagnosis of cognitive impairment, PTSD, anxiety, or depression, previous antipsychotic use.

3. Intervention characteristics-inhaled or intravenous agent used; indication for sedation (postoperative cardiac, postoperative non-cardiac, non-surgical or mixed), concentration (minimum alveolar concentration or end-tidal concentration for inhaled anaesthetics if available), primary versus adjunct mode of sedation, total duration of exposure to inhaled anaesthetic, mode of administration (ie, Anesthesia Conserving Device (AnaConDa) vs other).

4. Comparator characteristics-comparator(s) of interest include intravenous sedative used (eg, propofol, opioid, benzodiazepine, ketamine), dose of comparator(s) of interest used and age and sex of comparator group.

5. Outcomes-for each of cognitive function, anxiety, depression and PTSD: tool(s) of measurement (subjective vs objective, and the specific tool(s) used (ie, questionnaires, Mini-Mental State Examination, Trails A, etc for cognition; Hospital Anxiety and Depression or Patient Health Questionaire-9 for depression, etc or a battery of tests for each outcome)), definition of impairment (scores on objective testing), timing of measurement (eg, while in ICU, prior to hospital discharge; 3 months postdischarge, etc), rates of outcome of interest (eg, \% with depressive symptoms).

- For cognitive function only: domain of cognition affected (eg, executive function, language, etc).

- For depression, anxiety and PTSD only: rates of use of psychiatric medications (eg, antidepressants/anxiolytics, etc) in follow-up.

\section{Methodological appraisal}

Once we have selected studies for data extraction, each individual study will also be assessed for risk of bias based on publication type and validated screening tools. In order to assess the selected studies for bias, we will use the Cochrane risk of bias tool for any randomised controlled trials, and report individual domains of bias as either 'High' or 'Low' risk of bias. To assess biases in 
case-control and cohort studies, we will use the NewcastleOttawa Quality Assessment Scale. For any case series that are included, we will use the Joanna Briggs Institute's critical appraisal tool for case series.

\section{Data synthesis}

We will perform a narrative description of included fulltext studies for each of the four outcomes of cognition, depression, anxiety and PTSD. When available, we will present each individual outcome of interest in table format with a descriptive statistical focus with categorical variables (eg, sex, prevalence of quantitative impairment) expressed as a frequency of events, and quantitative variables from each study presented as medians. We do not anticipate a sufficient number of randomised controlled trials in this area to allow for a meta-analysis to be performed.

\section{Sensitivity analyses}

If possible, a sensitivity analysis will be performed on the indication for sedation (cardiac postoperative patients vs non-cardiac postoperative patients vs non-operative), as well as for duration of sedation (for more than 24 hours or less than 24 hours) in order to ascertain the effect of these variables on the outcome of interest.

\section{Patient and public involvement}

As this is a systematic review protocol, patients and the public were not directly involved in the formation of the protocol. Patient outcomes will be derived from original articles when published in the systematic review.

\section{Ethics and dissemination}

The systematic review uses published data and therefore does not require ethics approval. Results will be disseminated via publication in peer-reviewed journals and presentation at conferences related to the field.

\section{Author affiliations}

${ }^{1}$ Department of Medicine, University of Western Ontario, London, Ontario, Canada ${ }^{2}$ Department of Anesthesiology and Pain Medicine, Temetry Faculty of Medicine, University of Toronto, Toronto, Ontario, Canada

${ }^{3}$ Department of Pediatrics, Laval University, Quebec, Quebec, Canada

${ }^{4}$ Department of Anesthesiology, McGill University Faculty of Medicine, Montreal, Quebec, Canada

${ }^{5}$ Departments of Pharmacy and Medicine, Sinai Health System, Toronto, Ontario, Canada

${ }^{6}$ Leslie Dan Faculty of Pharmacy, University of Toronto, Toronto, Ontario, Canada ${ }^{7}$ Sunnybrook Research Institute, Department of Anesthesia, Sunnybrook Health Sciences Centre, Toronto, Ontario, Canada

${ }^{8}$ Institute of Health Policy, Mangement and Evaluation, University of Toronto, Toronto, Ontario, Canada

${ }^{9}$ Institute of Clinical Evaluative Sciences, Toronto, Ontario, Canada

${ }^{10}$ Department of Medicine, Western University, London, Ontario, Canada

\section{Twitter Conall Francoeur @Con_All}

Contributors MS and SC designed the project with assistance from AJ. SC drafted the initial manuscript. KG, CF, DW, LB and AJ all assisted in project design and writing of the manuscript. All authors edited and approved the final submission.

Funding The authors have not declared a specific grant for this research from any funding agency in the public, commercial or not-for-profit sectors.
Competing interests LB, AJ and MS have an ongoing clinical trial (ClinicalTrials. gov, NCT04415060) investigating the use of inhaled anaesthetics in the ICU.

Patient consent for publication Not required.

Provenance and peer review Not commissioned; externally peer reviewed.

Supplemental material This content has been supplied by the author(s). It has not been vetted by BMJ Publishing Group Limited (BMJ) and may not have been peer-reviewed. Any opinions or recommendations discussed are solely those of the author(s) and are not endorsed by BMJ. BMJ disclaims all liability and responsibility arising from any reliance placed on the content. Where the content includes any translated material, BMJ does not warrant the accuracy and reliability of the translations (including but not limited to local regulations, clinical guidelines, terminology, drug names and drug dosages), and is not responsible for any error and/or omissions arising from translation and adaptation or otherwise.

Open access This is an open access article distributed in accordance with the Creative Commons Attribution Non Commercial (CC BY-NC 4.0) license, which permits others to distribute, remix, adapt, build upon this work non-commercially, and license their derivative works on different terms, provided the original work is properly cited, appropriate credit is given, any changes made indicated, and the use is non-commercial. See: http://creativecommons.org/licenses/by-nc/4.0/.

\section{ORCID iDs}

Sean Cuninghame http://orcid.org/0000-0002-2594-6121

Kevin Gorsky http://orcid.org/0000-0002-8480-6516

\section{REFERENCES}

1 Kanji S, Burry L, Williamson D, et al. Therapeutic alternatives and strategies for drug conservation in the intensive care unit during times of drug shortage: a report of the Ontario COVID-19 ICU drug Task force. Can J Anaesth 2020;67:1405-16.

2 Jerath A, Ferguson ND, Cuthbertson B. Inhalational volatile-based sedation for COVID-19 pneumonia and ARDS. Intensive Care Med 2020;46:1-4.

3 Flinspach AN, Zacharowski K, loanna D, et al. Volatile isoflurane in critically ill coronavirus disease 2019 Patients-A case series and systematic review. Crit Care Explor 2020;2:e0256.

4 Hanidziar D, Bittner EA. Sedation of mechanically ventilated COVID-19 patients: challenges and special considerations. Anesth Analg 2020;131:e40-1.

5 Mesnil M, Capdevila X, Bringuier S, et al. Long-Term sedation in intensive care unit: a randomized comparison between inhaled sevoflurane and intravenous propofol or midazolam. Intensive Care Med 2011;37:933-41.

6 Jabaudon M, Boucher P, Imhoff E, et al. Sevoflurane for sedation in acute respiratory distress syndrome. A randomized controlled pilot study. Am J Respir Crit Care Med 2017;195:792-800.

7 Jerath A, Panckhurst J, Parotto M, et al. Safety and efficacy of volatile anesthetic agents compared with standard intravenous Midazolam/Propofol sedation in ventilated critical care patients: a meta-analysis and systematic review of prospective trials. Anesth Analg 2017;124:1190-9.

$8 \mathrm{MaX}$, Vervoort D. Critical care capacity during the COVID-19 pandemic: global availability of intensive care beds. J Crit Care 2020;58:96-7.

9 Burry LD, Barletta JF, Williamson D, et al. It takes a Village...: Contending with drug shortages during disasters. Chest 2020;158:2414-24.

10 Suleiman A, Qaswal AB, Alnouti M, et al. Sedating mechanically ventilated COVID-19 patients with volatile anesthetics: insights on the Last-Minute potential weapons. Sci Pharm 2021;89:6.

11 Honarmand K, Lalli RS, Priestap F, et al. Natural history of cognitive impairment in critical illness survivors. A systematic review. Am J Respir Crit Care Med 2020;202:193-201.

12 Kotfis K, Marra A, Ely EW. Icu delirium - a diagnostic and therapeutic challenge in the intensive care unit. Anestezjol Intens Ter 2018;50:160-7.

13 Wilson JE, Mart MF, Cunningham C, et al. Delirium. Nat Rev Dis Primers 2020;6:1-26.

14 Pandharipande PP, Girard TD, Jackson JC, et al. LongTerm cognitive impairment after critical illness. $N$ Engl J Med 2013;369:1306-16.

15 Müller A, von Hofen-Hohloch J, Mende M, et al. Long-Term cognitive impairment after ICU treatment: a prospective longitudinal cohort study (Cog-I-CU). Sci Rep 2020;10:1-10.

16 Sakusic A, O'Horo JC, Dziadzko M, et al. Potentially modifiable risk factors for long-term cognitive impairment after critical illness: a systematic review. Mayo Clin Proc 2018;93:68-82. 
17 Girard TD, Thompson JL, Pandharipande PP, et al. Clinical phenotypes of delirium during critical illness and severity of subsequent long-term cognitive impairment: a prospective cohort study. Lancet Respir Med 2018;6:213-22.

18 Nedergaard HK, Jensen HI, Stylsvig M, et al. Effect of Nonsedation on cognitive function in survivors of critical illness. Crit Care Med 2020;48:1790-8.

19 Rabiee A, Nikayin S, Hashem MD, et al. Depressive symptoms after critical illness: a systematic review and meta-analysis. Crit Care Med 2016;44:1744-53.

20 Nikayin S, Rabiee A, Hashem MD, et al. Anxiety symptoms in survivors of critical illness: a systematic review and meta-analysis. Gen Hosp Psychiatry 2016;43:23-9.

21 Righy C, Rosa RG, da Silva RTA, et al. Prevalence of post-traumatic stress disorder symptoms in adult critical care survivors: a systematic review and meta-analysis. Crit Care 2019;23:213.
22 Wade DM, Howell DC, Weinman JA, et al. Investigating risk factors for psychological morbidity three months after intensive care: a prospective cohort study. Crit Care 2012;16:R192.

23 Calvin CM, Batty GD, Der G, et al. Childhood intelligence in relation to major causes of death in 68 year follow-up: prospective population study. BMJ 2017;357:j2708.

24 Deary IJ, Strand S, Smith P, et al. Intelligence and educational achievement. Intelligence 2007;35:13-21.

25 Gale CR, Batty GD, Tynelius P. Intelligence in early adulthood and subsequent hospitalisation and admission rates for the whole range of mental disorders: longitudinal study of 1,049,663 men. Epidemiology 2010;21:70.

26 Kuncel NR, Hezlett SA. Fact and fiction in cognitive ability testing for admissions and hiring decisions. Curr Dir Psychol Sci 2010;19:339-45.

27 Rethlefsen ML, Kirtey S, Waffenschmidt S, et al. PRISMA-S: an extension to the PRISMA statement for reporting literature searches in systematic reviews 2021;10:1-19. 